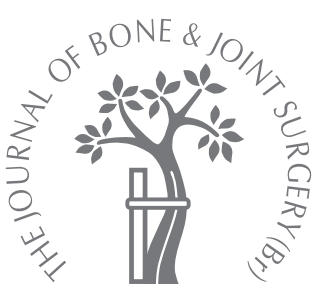

M. Lampasi, C. Bettuzzi, M. Palmonari, O. Donzelli

From Rizzoli Orthopaedic Institute, Bologna, Italy

\title{
Transfer of the tendon of tibialis anterior in relapsed congenital clubfoot
}

\author{
LONG-TERM RESULTS IN 38 FEET
}

A total of 38 relapsed congenital clubfeet (16 stiff, 22 partially correctable) underwent revision of soft-tissue surgery, with or without a bony procedure, and transfer of the tendon of tibialis anterior at a mean age of 4.8 years $(2.0$ to 10.1$)$. The tendon was transferred to the third cuneiform in five cases, to the base of the third metatarsal in ten and to the base of the fourth in 23. The patients were reviewed at a mean follow-up of 24.8 years (10.8 to 35.6). A total of 11 feet were regarded as failures (one a tendon failure, five with a subtalar fusion due to over-correction, and five with a triple arthrodesis due to under-correction or relapse).

In the remaining feet the clinical outcome was excellent or good in $\mathbf{2 0}$ and fair or poor in seven. The mean Laaveg-Ponseti score was 81.6 of 100 points (52 to 92). Stiffness was mild in four feet and moderate or severe in 23.

Comparison between the post-operative and follow-up radiographs showed statistically significant variations of the talo-first metatarsal angle towards abduction. Variations of the talo-calcaneal angles and of the overlap ratio were not significant.

Extensive surgery for relapsed clubfoot has a high rate of poor long-term results. The addition of transfer of the tendon of tibialis anterior can restore balance and may provide some improvement of forefoot adduction. However, it has a considerable complication rate, including failure of transfer, over-correction, and weakening of dorsiflexion. The procedure should be reserved for those limited cases in which muscle imbalance is a causative or contributing factor.

There is no standard operation for relapsed clubfoot because different deformities may co-exist. The various soft-tissue and bony procedures available are mostly directed at correction of specific anatomic deformities, and operations may therefore vary between cases. ${ }^{1-3}$

Transfer of the tendon of tibialis anterior for the treatment of recurrent congenital clubfoot was described by Garceau ${ }^{4}$ in 1940, and has become popular for the treatment of relapsing clubfoot as a part of the Ponseti method. ${ }^{5-9}$

The purpose of this study was to evaluate the contribution of this operation to other corrective procedures and to review the long-term results in feet treated with revision operations which included this transfer.

to Dr M. Lampasi; e-mail: manuele.lampasi@hotmail.it

(C)2010 British Editorial Society of Bone and Joint Surgery doi:10.1302/0301-620X.92B2. $22504 \$ 2.00$

$J$ Bone Joint Surg $[\mathrm{Br}]$ 2010;92-B:277-83. Received 24 February 2009; Accepted after revision 25 September 2009 clinical and radiographic review. There were 11 bilateral cases, resulting overall in 38 feet (22 left, 16 right) for assessment.

Patient data included their age at presentation, the treatment before relapse and grading of the deformity (Manes, Costa and Innao ${ }^{10}$ ), stiffness of the foot, the clinical strength of the peroneal muscles, pre-operative electrophysiology, procedures associated with transfer of the tendon of tibialis anterior and the surgical technique of tendon transfer.

Evaluation at follow-up comprised a clinical and radiographic examination, the clinical and functional outcome, complications and any further surgery.

The appearance of the feet at follow-up was classified according to Garceau and Palmer, ${ }^{11}$ based on residual metatarsus adductus, heel varus and equinus. The clinical outcome was evaluated by the Laaveg-Ponseti ${ }^{12}$ system (excellent result, 90 to 100 points; good, 80 to 89; fair, 70 to 79 ; poor, $<70)$, based on pain, function, personal satisfaction, the range of movement of the ankle and foot, alignment of the hindfoot and forefoot, and walking ability. 


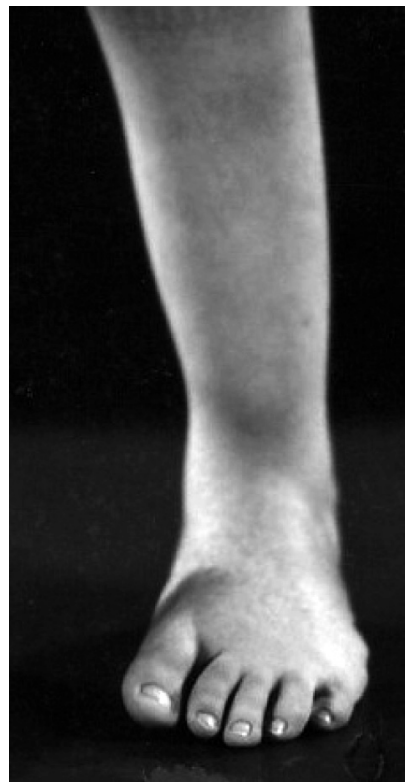

Fig. 1a

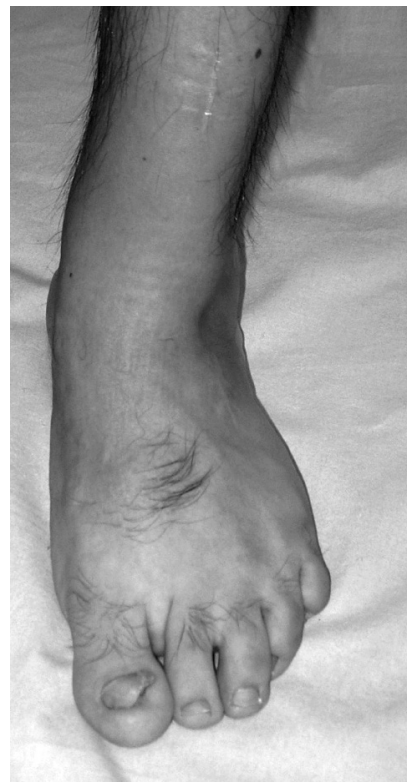

Fig. 1b
Photograph of a) a 3.5-year-old boy presenting with a left relapsed clubfoot. A posterior and medial release had been performed at the age of two months. There was weakness of the peroneal muscles, but electrophysiology was normal. Medial release combined with tibialis anterior tendon transfer to the base of the fourth metatarsal was performed. b) Clinical appearance at the age of 28 years and ten months. The foot showed an 'excellent' appearance ${ }^{11}$ and normal active dorsiflexion. Nevertheless, the clinical outcome ${ }^{12}$ was poor (60 of 100).

Stiffness was evaluated according to Garceau and Palmer, ${ }^{11}$ assessing the mobility of the peritalar joint from zero (normal) to four (a triple arthrodesis).

The motor strength of the transferred tibialis anterior muscle was assessed according to Jones' 0 to 5 -point scale. ${ }^{13}$

Weight-bearing radiographs of the foot were taken and the talo-calcaneal angle, the talo-first metatarsal angle on the AP view, and the talo-calcaneal angle and the first-fifth metatarsal overlap ratio on the lateral view were measured. The overlap ratio, ${ }^{7}$ was used as an indication of the degree of supination of the forefoot. The measurements were performed by three independent observers (ML, CB and MP) and the mean value of the angles and the median value of the overlap ratio were used.

Where post-operative radiographs carried out weightbearing three to six months after operation were available, the same measurements were performed on these films and comparison made between the post-operative and follow-up values.

The choice of comparison among these radiographic values was based on the assumption that variations between pre- and post-operative examination might be ascribed to the operation, whereas eventual differences, particularly as to eversion/abduction, might be attributed to the dynamic action of the transferred tibialis anterior.
Table I. Details of operations performed

\begin{tabular}{lc}
\hline & Number of feet \\
\hline Age at surgery (yrs) & 15 \\
2 to 3 & 14 \\
4 to 5 & 5 \\
6 to 8 & 4 \\
9 to 12 & \\
& \\
Site of re-insertion & 5 \\
3rd cuneiform & 10 \\
3rd metatarsal & 23 \\
4th metatarsal & \\
& \\
Procedures associated with TATT & \\
Post & 2 \\
Med & 11 \\
Post + Med & 8 \\
Post + Lat & 1 \\
Post + Med + Lat & 5 \\
Med + Lat & 8 \\
Med + Lat + PB & 3 \\
\hline * TAT, tibialis anterior tendon transfer excluding other \\
associated procedures, three cases of calcaneal osteotomy, \\
four tibial osteotomies and one osteotomy of the first \\
metatarsal; Post, posterior release; Med, medial release; Lat, \\
shortening of the lateral column; PB, tensioning of peroneus \\
brevis tendon \\
\end{tabular}

Statistical analysis. This was performed on these data using the $t$-test method. A p-value of $<0.05$ was considered significant.

\section{Results}

Revision surgery including transfer of the tibialis anterior was performed at a mean age of four years and ten months ( 2 years to 10 years and 2 months). The deformity was stiff in 16 feet and partially correctable in 22 . In no case was the deformity dynamic or flexible. Severity ${ }^{10}$ was categorised as grade I (reducibility of varus, equinus or supination possible up to $<20^{\circ}$ ) in ten feet, grade II (reducibility up to $20^{\circ}$ to $45^{\circ}$ ) in 22 feet and grade III (reducibility up to $45^{\circ}$ ) in six feet. Before revision surgery, a mean of 1.1 operations had been performed, in which 34 feet underwent one operation and four had two operations. In these 42 operations, a mean of 1.8 surgical procedures per foot (one to four) was performed: a posterior release was performed in all cases, associated with a medial release in 26 of these. In no case had the Ponseti technique ${ }^{12}$ been used.

At clinical examination, the strength of the peroneal muscles was assessed as normal in 26 patients and weak in 12 (Fig. 1). After ethical approval and informed consent, these 12 underwent electromyography as described by Costa, Gualtieri and Merlini. ${ }^{14}$ Normal findings were recorded in all cases.

There was no pre-operative casting. 


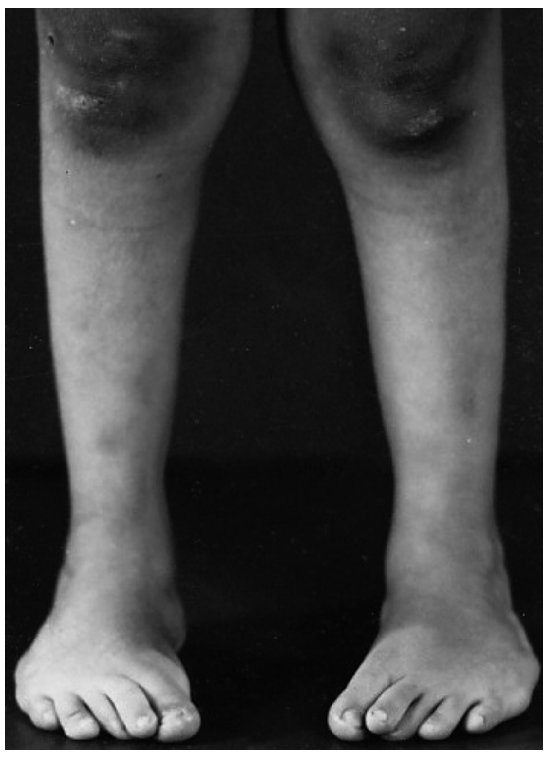

Fig. 2a

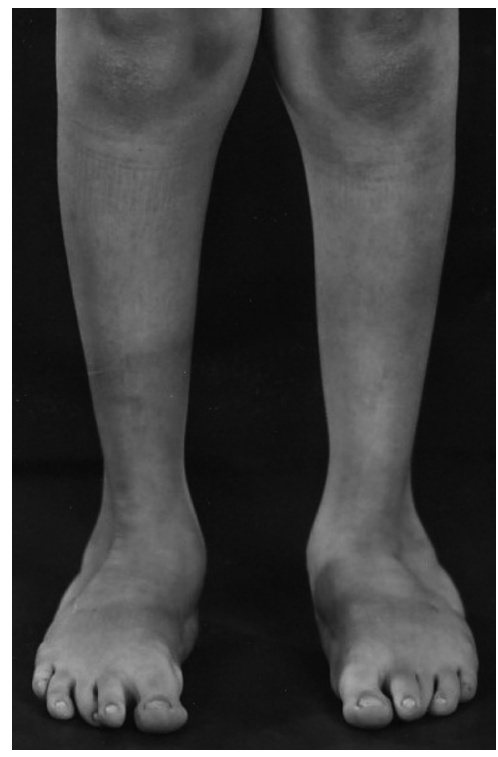

Fig. $2 b$

Photograph of a) a 5.5-year-old boy presenting with bilateral relapsed clubfoot (a posterior and medial release had been performed at the age of four months). Both feet underwent medial release and tibialis anterior tendon transfer to the base of the fourth metatarsal in the right foot and to the third cuneiform on the left. b) At age seven years and eight months both feet show over-correction with a valgus pronated flatfoot. Subtalar arthrodesis and re-insertion of the tendon into its anatomical site was performed bilaterally.

The operations to correct the deformity, along with transfer of the tendon of tibialis anterior, are summarised in Table I. Medial release was performed in 35 feet (92\%) and posterior release in $16(42 \%)$, whereas bony procedures to shorten the lateral column were undertaken in 17 feet $(45 \%)$, including 12 cases of distal intra-articular resection of the calcaneum ${ }^{15}$ and five of calcaneocuboid joint excision and fusion. ${ }^{16}$

Transfer of the tendon of tibialis anterior was performed according to the technique of Garceau. ${ }^{4}$ Three incisions were made, one to detach the tendon from its insertion, a second at the musculotendinous junction, through which the tendon was retrieved before moving it laterally and a third to reinsert the tendon to the base of the third metatarsal in ten cases, to the third cuneiform in five and to the base of the fourth metatarsal in 23 . The site of reinsertion depended on the severity of the deformity, the correction achieved by the associated procedures, and the preferences of the five different surgeons (OD, SS, RL, LV, ADM) who performed the operations.

The tendon was transferred under the extensor retinaculum in 24 feet and superficial to it in a further 14. A hole was drilled through the site for reinsertion. The tendon was prepared with a resorbable suture, and then passed through the hole, withdrawn from the plantar surface and sutured under tension. The tendon was also sutured to the periosteum. A below-knee cast was applied for four weeks, followed by a weight-bearing cast for a further four weeks. There were no wound complications.
The patients were re-evaluated at a mean time from revision of 24.8 years (10.8 to 35.6$)$.

One foot required re-operation 2.9 years after transfer of the tendon of tibialis anterior owing to failure of the tendon transfer at the site of reinsertion. The surgeon decided to reinsert the tendon into its anatomical site. Five feet $(13.2 \%)$ had undergone a Grice subtalar fusion with reinsertion of the tendon into its anatomical site after a mean of four years (2.2 to 7.7$)$ after transfer of tibialis anterior because of over-correction of the foot (Fig. 2). Five feet had undergone triple arthrodesis, combined with calcaneal osteotomy in one case, at a mean of 10.4 years (7.8 to 17.6 ) because of under-correction or relapse of the deformity. All these 11 cases $(28.9 \%)$ were regarded as failures of the revision and were excluded from the final evaluation of the clinical outcome.

Conversely, three feet had undergone surgery for reinsertion of the tendon into the anatomical site at a mean of 4.4 years (3.0 to 5.8) after transfer of the tendon of tibialis anterior, owing to early evidence of over-correction in a well-corrected foot. These feet were included in the final clinical follow-up.

A total of 27 feet underwent clinical examination at a mean of 23.5 years (10.8 to 35.6) after operation. All patients had reached skeletal maturity, with a mean age at follow-up of 28 years (15.0 to 42.7) (Table II). The clinical outcome was excellent in four feet, good in 16, fair in five and poor in two. The mean Laaveg-Ponseti ${ }^{12}$ score was 81.6 (52 to 92 ) points. The more common subjective com- 
Table II. Clinical outcome at follow-up 27 of 38 feet examined $)^{*}$

\begin{tabular}{lc}
\hline & Number of feet \\
\hline Clinical outcome $^{12}$ & \\
Excellent & 4 \\
Good & 16 \\
Fair & 5 \\
Poor & 2 \\
& \\
Appearance & \\
Excellent & \\
Good & 7 \\
Fair & 17 \\
Poor & 3 \\
& 0
\end{tabular}

Motor strength of transferred tibialis anterior

$\begin{array}{rr}5 / 5 & 22 \\ 4 / 5 & 5 \\ <3 / 5 & 0\end{array}$

Stiffness ${ }^{11}$

$0 / 4 \quad 0$

$1 / 4$

$2 / 4$

$3 / 4$

$4 / 4$

\section{5}

* 11 of 38 feet $(28.9 \%)$ were regarded as failures and excluded due to tendon failure, subtalar arthrodesis or triple arthrodesis before follow-up

plaints were of pain on excessive exercise, scars, a thin calf and stiffness. The appearance ${ }^{11}$ was excellent in seven cases, good in 17 and fair in three. Stiffness ${ }^{11}$ was mild in four feet, moderate in 15 and severe in eight.

The mean passive dorsiflexion of the ankle was to $13.4^{\circ}$ $\left(0^{\circ}\right.$ to $\left.25^{\circ}\right)$. Eight feet showed dorsiflexion of $\leq 5^{\circ}$, eight $6^{\circ}$ to $15^{\circ}$, and 11 were $>15^{\circ}$. There was no significant correlation between dorsiflexion and outcome.

In one foot where the tendon was transferred over the extensor retinaculum, the 'bowstring' phenomenon ${ }^{5,8}$ was evident (Fig. 3).

The motor strength ${ }^{13}$ of the transferred tibialis anterior was normal (5 of 5) in 22 feet and good ( 4 of 5) in five, including the foot with the bowstring phenomenon.

There was no statistically significant correlation between the clinical features at presentation, the site of reinsertion of the tendon and the clinical outcome $(\mathrm{p}>0.05)$.

With the three feet that required reinsertion of the tendon into its anatomical site, forefoot abduction did not progress and over-correction did not develop. The strength of the retransferred tendon was 4 of 5 in one case and 5 of 5 in two.

Radiographic evaluation, including post-operative and follow-up of radiographs, was available for 14 feet with a mean follow-up of 16.1 years ( 3 to 32 ), including 13 transfers along the fourth and one along the third metatarsal axis. Of these, three feet had required subtalar fusion due to over-correction and three re-insertion of the tendon into its anatomical site. In these cases measurements were made on radiographs taken before the latter procedure. The mean angle between the talus and the first metatarsal on the AP view was $\mathrm{a}+17.5^{\circ}\left(-15^{\circ}\right.$ to $\left.46^{\circ}\right)$ in the post-operative films and $+1.6^{\circ}\left(-18^{\circ}\right.$ to $\left.30^{\circ}\right)$ at follow-up. Statistical analysis using $t$-tests was applied to the variations of this angle and found to be statistically significant $(\mathrm{p}<0.05)$, which was indicative of progressive forefoot abduction. The mean variation was greater in more flexible $\left(-19^{\circ},-3^{\circ}\right.$ to $\left.-41^{\circ}\right)$ than in more rigid feet $\left(-10.2^{\circ},-2^{\circ}\right.$ to $\left.-18^{\circ}\right)$, but it was not possible to perform statistical tests to look for correlation of this variation with the pre-operative flexibility of the foot, given the small number of cases.

There was no statistically significant $(\mathrm{p}>0.0 .5)$ variation between the post-operative and follow-up measurements of the talo-calcaneal angles in the AP view (mean variation $-0.6^{\circ}\left(-16^{\circ}\right.$ to $\left.\left.12^{\circ}\right)\right)$ and lateral views (mean variation $-0.9^{\circ}\left(-9^{\circ}\right.$ to $\left.13^{\circ}\right)$, and in the overlap ratio (mean variation -0.7 points, $(-4$ to 1$)$.

\section{Discussion}

Relapsed clubfoot deformity may be due to incomplete primary correction, loss of reduction, scarring, ${ }^{2,17-19}$ muscle imbalance or the original pathology. $8,9,15,20$

As different deformities may be present, a comprehensive surgical approach is required, tailored to the deformity $2,6,21,22$ and the age of the child. ${ }^{19}$ Surgical techniques for relapsed congenital clubfeet can be divided into three broad categories namely soft-tissue releases, bony procedures and tendon transfers, the former two representing the main corrective procedures. ${ }^{16}$

In the younger child soft-tissue release may be sufficient to achieve correction which has not been provided by primary treatment, ${ }^{16,20}$ whereas bony operations, variously combined with soft-tissue procedures, are required in the older child. ${ }^{19}$

Tendon transfers play a secondary role and are indicated when muscle imbalance is a deforming or aggravating factor. ${ }^{2,16,23-27}$ Such imbalance may occur with a range of presentations. That between invertor and evertor muscles ${ }^{6}$ is common after primary treatment of congenital clubfoot. Children have feet that supinate during the swing phase and are supinated to some extent in the stance phase, but which are fully correctable at static examination. ${ }^{6,9}$ In most cases spontaneous correction occurs with maturation of muscle function. ${ }^{6,7}$

Where dynamic supination persists, it may lead to progressive and persistent clubfoot deformity as demonstrated by experimental studies ${ }^{28}$ where fixed supination deformity developed after dividing the peroneal tendons. In some of these feet muscle or nerve dysfunction may be shown by electrophysiological studies. ${ }^{6,26}$

Conversely, imbalance may also be secondary to anatomical factors, such as abnormal insertion of the tibialis anterior tendon, or to the deformity itself. Incomplete primary reduction of the medially displaced navicular may result in a supinating force by the tibialis anterior tendon and the 


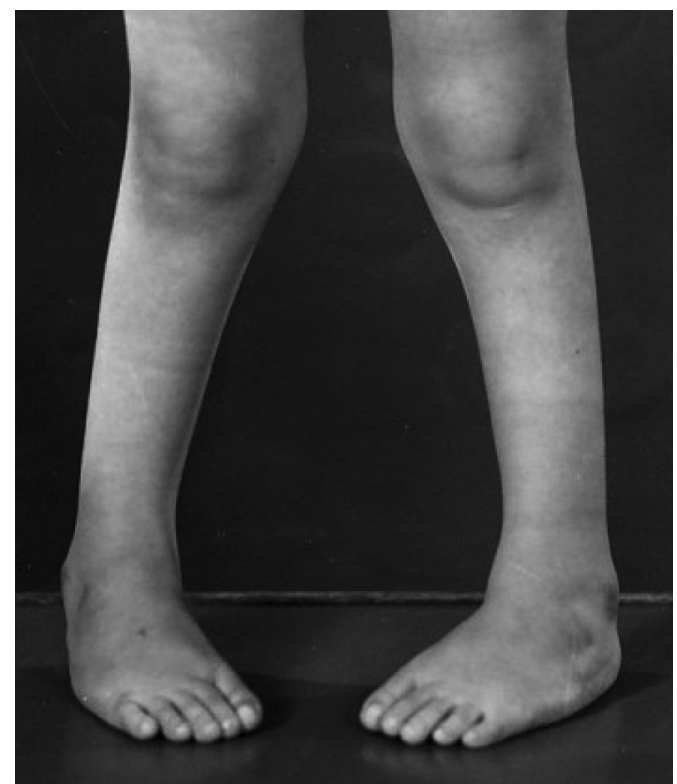

Fig. 3a

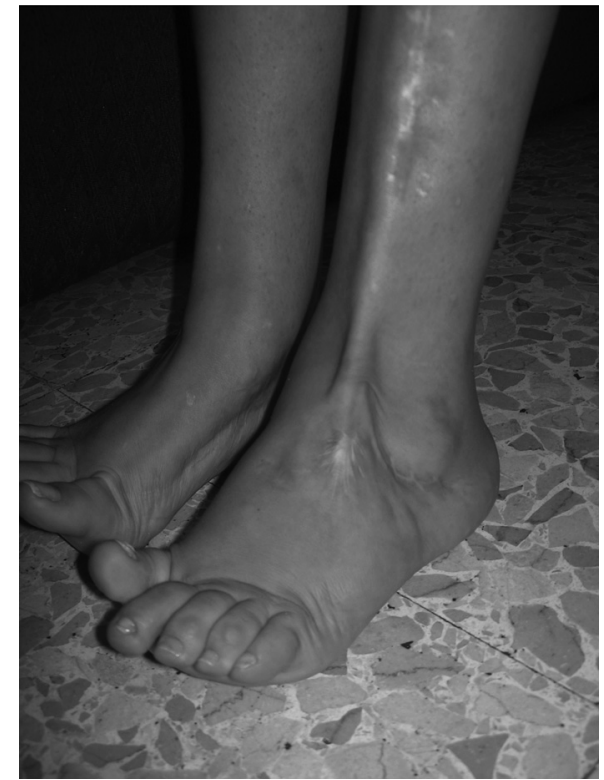

Fig. 3b

Photograph of a) a 4.3-year-old girl presenting with a bilateral relapsed clubfoot. She had already undergone a bilateral posteromedial release at the age of three months and re-operation on the left foot at the age of 2.3 years. Revision surgery on both feet included a medial release, a distal intra-articular resection of the calcaneum ${ }^{15}$ and tibialis anterior tendon transfer over the extensor retinaculum and reinsertion at the base of the fourth metatarsal. b) Clinical appearance at the age of 21 years and eight months. The right foot showed an excellent clinical outcome (score 90 of 100), ${ }^{12}$ a 'good' appearance, ${ }^{11}$ moderate (2 of 4) stiffness, ${ }^{11}$ and normal strength of the transferred tibialis anterior. The left foot showed a fair clinical outcome (score 76 of 100), a 'good' appearance ${ }^{11}$ and severe (3 or 4) stiffness. ${ }^{11}$ On active dorsiflexion the 'bowstring' phenomenon was evident, which caused an aesthetic complaint and reduction of the strength of tibialis anterior (score: 4 or 5).

mechanical disadvantage produced by the deformity ${ }^{11,29}$ may often cause a relative weakness of peroneal muscles. ${ }^{6,25,26}$ In such cases, once the deformity is corrected the peroneal muscles may regain function and muscle balance will be restored.

The transfer of the tendon of tibialis anterior is an important part of the Ponseti method for the treatment of relapsing clubfeet ${ }^{5-9,30}$ and is shown to be a reasonable means of achieving balance and preventing the progression of deformity. ${ }^{7,8}$ In these cases, this transfer, mostly performed as an isolated procedure, requires early recognition of recurrent deformity $^{9}$ and a flexible foot..$^{5,8,21}$

In the presence of joint contractures or bony deformities, the transfer is associated with various possible revision procedures, which are based mainly on clinical and radiographic examination but also on intra-operative findings. Gait analysis may be useful to characterise the deformity, particularly in detecting an overactive tibialis anterior using dynamic electromyography. ${ }^{31}$ Electrophysiological examination may also be helpful to detect muscle or nerve dysfunction in cases with severe imbalance.

Our review is an analysis of the long-term results of different and complex revision procedures which included transfer of the tendon of tibialis anterior, and shows the usual limitation of this kind of study, in that there are many variables. However, our results concur with previous reports in that surgery for relapsed clubfoot gives a high percentage of unsatisfactory results. ${ }^{19,32}$ Unlike other authors', ${ }^{12}$ we found no significant correlation between passive ankle dorsiflexion and outcome.

In our evaluation of the specific contribution of transfer of the tendon of tibialis anterior we compared postoperative and follow-up angles, which is a method not reported in other studies. In our opinion, comparisons between pre-operative and follow-up examinations ${ }^{7,8}$ are inadequate if additional procedures are associated with the transfer. Even though our radiographic evaluation has the bias of a high percentage of over-corrected cases, our results show that the transfer of the tendon of tibialis anterior mainly acts by providing a progressive reduction of the talo-first metatarsal angle, with a greater reduction in more correctable feet. With regard to the reproducibility of measurement of the talo-first metatarsal angle, it has been considered reproducible by some researchers ${ }^{33}$ whereas others $^{34}$ found high intra- and interobserver variations. Conversely, in our study, improvement of supination and of the talo-calcaneal angles was not significant.

Other authors have reported similar but greater variations, namely a significant correction of forefoot adduction, ${ }^{7,8}$ improvement in the overlap ratio $^{7}$ and an increase in the talocalcaneal angles in AP and lateral views, ${ }^{5,35}$ but 
have either shown the above-mentioned limitations or that the procedure was performed on flexible feet.

In summary, in feet with a rigid or partially correctable deformity transfer of the tendon of tibialis anterior should not be expected to provide much improvement, except for reduction of adduction in more flexible feet, and correction should be provided by other procedures.

With regard to the site of reinsertion, the biomechanical effect of different sites has been studied by Hui, Goh and Lee $^{36}$ in cadavers. The ideal site for full tendon transfer was found to be along the axis of the third metatarsal, and insertion lateral to this caused greater eversion. However, the normal cadaver feet in their study might mimic flexible feet requiring muscle balance, but not relapsed clubfeet. In our radiographic analysis, 13 of 14 transfers were along the fourth metatarsal axis, so that a statistical study of correlation between the site of reinsertion and radiographic variations was not feasible.

Currently, our aim when seeking complete correction of the deformity or more flexible feet, is to reinsert the tendon along the third metatarsal axis. A more lateral insertion along the line of the fourth metatarsal may be needed in feet with imbalance and severe deformities, or greater risk of relapse, such as those that have had many operations or have much scar tissue.

In our study we did not specifically analyse the effect of transfer of the tendon of tibialis anterior on muscle balancing. The mid-term results, at a mean follow-up of 5.2 years ( 2 to 12.5 ), were evaluated in a recent paper $^{27}$ with a subjective scoring system of restoration of balance. Good results were reported in $87 \%$ to $88 \%$ of feet treated for dynamic supination only or for dynamic supination with other structural deformities, and fair results were reported in $12 \%$ to $13 \%$ of cases.

With regard to complications, over-correction is the most troublesome. Whereas a small degree of under-correction is often well tolerated in adulthood, over-correction is less acceptable and difficult to treat. ${ }^{20,37}$ Transferring the tendon to the cuboid or base of the fifth metatarsal ${ }^{4,26,38}$ has resulted in a high rate of over-correction and should be avoided. Even in our experience of reinsertion along the third and fourth metatarsal axis, there was a high rate of over-correction ( 5 of 38 feet, 13.1\%) with no significant correlation with the site of re-insertion, the age at operation or the clinical features. It is possible that muscle imbalance was overestimated in these feet.

Careful and frequent follow-up is advised. If any tendency to eversion develops, the tendon should be returned to the medial aspect of the foot. ${ }^{25} \mathrm{~A}$ split transfer has been proposed as an alternative to complete transfer of the tendon of tibialis anterior in order to avoid this complication. . $^{21}$

Another risk is to weaken the strength of dorsiflexion. Five of our cases had reduced strength in the transferred tendon. This was found particularly in one of the three feet in which the tendon was returned to its anatomical site, and in the case with the 'bowstring' phenomenon. ${ }^{5,8}$ For this reason, we currently transfer the tendon beneath the extensor retinaculum.

Extensive surgery for clubfoot has a high rate of poor results, stiffness, failures and re-operations, and transfer of the tendon of tibialis anterior adds its own problems. The role of this procedure for relapsed clubfoot should be reserved for those limited cases where muscle imbalance is a causative or contributing factor. In particular, the surgeon should confirm whether muscle imbalance is due to dysfunction between invertors and evertors, a mechanical consequence of the deformity, or both. If the transfer is required, a careful technique should be used, particularly in placing the tendon beneath the extensor retinaculum, and choosing the site and fixation of the transfer.

No benefits in any form have been received or will be received from a commer cial party related directly or indirectly to the subject of this article.

\section{References}

1. Kose N, Günal I, Göktürk E, Seber S. Treatment of severe residual clubfoot deformity by trans-midtarsal osteotomy. J Pediatr Orthop B 1999;8:251-6.

2. Vizkelety T, Szepesi K. Reoperation in treatment of clubfoot. J Pediatr Orthop 1989;9:144-7.

3. Bensahel H, Csukonyi Z, Desgrippes Y, Chaumien JP. Surgery in residual clubfoot: one-stage medioposterior release "a la carte". J Pediatr Orthop 1987;7:145-8.

4. Garceau GJ. Anterior tibial tendon transposition in recurrent congenital club-foot. $J$ Bone Joint Surg 1940;22:932-6.

5. Ippolito E, Ricciardi-Pollini PT, Tudisco C, Ronconi P. The treatment of relapsing clubfoot by tibialis anterior transfer underneath the extensor retinaculum. Ital $J$ Orthop Traumato/ 1985;11:171-7.

6. Ezra E, Hayek S, Gilai AN, Khermosh $\mathbf{0}$, Wientroub $\mathbf{S}$. Tibialis anterior tendon transfer for residual dynamic supination deformity in treated club feet. $J$ Pediatr Orthop B 2000;9:207-11.

7. Kuo KN, Hennigan SP, Hastings ME. Anterior tibial tendon transfer in residual dynamic clubfoot deformity. J Pediatr Orthop 2001;21:35-41.

8. Farsetti P, Caterini R, Mancini F, Potenza V, Ippolito E. Anterior tibial tendon transfer in relapsing congenital clubfoot: long-term follow-up study of two series treated with a different protocol. J Pediatr Orthop 2006;26:83-90.

9. Dietz FR. Treatment of a recurrent clubfoot deformity after initial correction with the Ponseti technique. Instr Course Lect 2006;55:625-9.

10. Manes E, Costa CM, Innao V. Treatment of congenital club-foot during the 1 st years of life. Chir Organi Mov 1975;62:301-14 (in Italian).

11. Garceau GJ, Palmer RM. Transfer of the anterior tibial tendon for recurrent club foot: a long-term follow-up. J Bone Joint Surg [Am] 1967;49-A:207-31.

12. Laaveg SJ, Ponseti IV. Long-term results of treatment of congenital club foot. $J$ Bone Joint Surg [Am] 1980;62-A:23-31.

13. Herring JA. Tachdjian's paediatric orthopaedics. Fourth ed. Philadelphia: Saunders, 2008:48.

14. Costa C, Gualtieri G, Merlini L. Electromyographic findings in congenital clubfoot. Chir Organi Mov 1978;74:415-17 (in Italian).

15. Lichtblau S. A medial and lateral release operation for club foot: a preliminary report. J Bone Joint Surg [Am] 1973;55-A:1377-84.

16. Evans D. Relapsed club foot. J Bone Joint Surg [Br] 1961;43-B:722-33.

17. Tanaf YN, Carroll NC. Analysis of the components of residual deformity in clubfeet presenting for reoperation. J Pediatr Orthop 1992;12:207-16.

18. Crawford AH, Gupta AK. Clubfoot controversies: complications and causes for failure. Instr Course Lect 1996;45:339-46.

19. Harvey AR, Uglow MG, Clarke NM. Clinical and functional outcome of relapse surgery in severe congenital talipes equinovarus. J Pediatr Orthop B 2003;12:49-55.

20. Park SS, Kim SW, Jung BS, Lee JS, Kim JS. Selective soft-tissue for recurrent or residual deformity after conservative treatment of idiopathic clubfoot. J Bone Joint Surg [Br] 2009;91-B:1526-30.

21. Kuo KN, Smith PA. Correcting residual deformity following clubfoot releases. Clin Orthop 2009;467:1326-33.

22. Mehrashan M, Rampal V, Seringe R, Wicard P. Recurrent club-foot deformity following previous soft-tissue release: mid-term outcome after revision surgery. J Bone Joint Surg [Br] 2009;91-B:949-54

THE JOURNAL OF BONE AND JOINT SURGERY 
23. Singer $\mathbf{M}$, Fripp AT. Tibialis anterior transfer in congenital club foot. J Bone Joint Surg [Br] 1958;40-B:252-5.

24. Turco VJ. Clubfoot: current problems in orthopaedics. First ed. New York: ChurchillLivingstone, 1981:159.

25. Huang YT, Lei W, Zhao L, Wang J. The treatment of congenital club foot by operation to correct deformity and achieve dynamic muscle balance. J Bone Joint Surg [Br] 1999:81-B:858-62

26. Feldbrin Z, Gilai AN, Ezra E, et al. Muscle imbalance in the aetiology of idiopathic club foot: an electromyographic study. J Bone Joint Surg [Br] 1995;77-B:596-601.

27. Thompson GH, Hoyen HA, Barthel T. Tibialis anterior tendon transfer after clubfoot surgery. Clin Orthop 2009;467:1306-13.

28. Langenskiöld A, Ritsilä V. Supination deformity of the forefoot. Acta Orthop Scand 1977;48:325-33.

29. Critchley JE, Taylor RG. Transfer of the tibialis anterior tendon for relapsed clubfoot. J Bone Joint Surg [Br] 1952;34-B:49-52.

30. Cooper DM, Dietz FR. Treatment of idiopathic clubfoot: a thirty-year follow-up note. J Bone Joint Surg [Am] 1995;77-A:1477-89.
31. Sankar WN, Rethlefsen SA, Weiss J, Kay RM. The recurrent clubfoot: can gait analysis help us to make better preoperative decisions? Clin Orthop 2009;467:1214-22.

32. Dobbs MB, Nunley R, Schoenecker PL. Long-term follow-up of patients with clubfeet treated with extensive soft-tissue release. J Bone Joint Surg [Am] 2006;88A:986-96

33. Fridman MW, de Almeida Fialho HS. The role of radiographic measurements in the evaluation of congenital clubfoot surgical results. Skeletal Radio/2007;36:129-38.

34. Herbsthofer B, Eckardt A, Rompe JD, Küllmer K. Significance of radiographic angle measurements in evaluation of congenital clubfoot. Arch Orthop Trauma Surg 1998;117:324-9

35. Ricciardi-Pollini PT, Ippolito E, Tudisco C, Farsetti P. Congenital clubfoot: results of treatment of 54 cases. Foot Ankle 1984;5:107-17.

36. Hui JH, Goh JC, Lee EH. Biomechanical study of tibialis anterior tendon transfer. Clin Orthop 1998;349:249-55.

37. Besse JL, Leemrijse T, Themar-Noel C, et al. Congenital club foot: treatment in childhood, outcome and problems in adulthood. Rev Chir Orthop Reparatrice Appar Mot 2006;92:175-92 (in French).

38. Esser RD. The medial sagittal approach in the treatment of the congenital clubfoot: a follow-up report of a 15-year experience. Clin Orthop 1994;302:156-63. 